\title{
Combined effect of hypothermia and caspase-2 gene deficiency on neonatal hypoxic-ischemic brain injury
}

\author{
Ylva Carlsson ${ }^{1,2}$, Xiaoyang Wang ${ }^{1}$, Leslie Schwendimann ${ }^{3}$, Catherine I. Rousset ${ }^{1,4}$, Etienne Jacotot ${ }^{4,5}$, Pierre Gressens ${ }^{3,4}$, \\ Marianne Thoresen ${ }^{6,7}$, Carina Mallard ${ }^{1}$ and Henrik Hagberg ${ }^{1,2,4}$
}

INTODUCTION: Hypoxia-ischemia (HI) injury in term infants develops with a delay during the recovery phase, opening up a therapeutic window after the insult. Hypothermia is currently an established neuroprotective treatment in newborns with neonatal encephalopathy (NE), saving one in nine infants from developing neurological deficits. Caspase-2 is an initiator caspase, a key enzyme in the route to destruction and, therefore, theoretically a potential target for a pharmaceutical strategy to prevent HI brain damage.

METHODS: The aim of this study was to explore the neuroprotective efficacy of hypothermia in combination with caspase-2 gene deficiency using the neonatal Rice-Vannucci model of HI injury in mice.

RESULTS: $H$ I brain injury was moderately reduced in caspase-2-1mice as compared with wild-type (WT) mice. Five hours of hypothermia $\left(33^{\circ} \mathrm{C}\right)$ vs. normothermia $\left(36^{\circ} \mathrm{C}\right)$ directly after $\mathrm{HI}$ provided additive protection overall (temperature $P=0.0004$, caspase- 2 genotype $P=0.0029$ ), in the hippocampus and thalamus, but not in other gray matter regions or white matter. Delayed hypothermia initiated $2 \mathrm{~h}$ after $\mathrm{HI}$ in combination with caspase-2 gene deficiency reduced injury in the hippocampus, but not in other brain areas.

DISCUSSION: In conclusion, caspase-2 gene deficiency combined with hypothermia provided enhanced neuroprotection as compared with hypothermia alone.

N eonatal encephalopathy (NE) at term affects $\sim 1-2 / 1,000$ born infants, and hypoxia-ischemia (HI) plays an important part in its etiology (1). HI brain injury develops with a delay during the recovery phase, opening up a therapeutic window after the insult (2). Clinical trials show that brain cooling after perinatal asphyxia reduces cerebral injury and improves outcome in term infants (3). Hypothermia is advised by the international resuscitation council as standard of care after moderate or severe perinatal HI brain injury. However, hypothermia reduces the incidence of poor outcome of death or disability only from $66 \%$ to $50 \%$ (ref. 4 ). Therefore, we seek to develop supplementary strategies of neuroprotection to add to currently applied hypothermic regimes in term infants with NE.

There is considerable support for apoptotic mechanisms being critical for the development of neonatal HI brain injury; more so than in the adult brain (2). Cysteine-dependent aspartate-directed protease (caspase)-dependent pathways are activated to execute apoptosis in the immature brain in response to HI $(5,6)$. Pan-caspase inhibition has been shown to reduce neonatal brain injury after $\mathrm{HI}$ in some models (6). However, as they are nonselective and caspases are important for normal brain development, a more specific inhibition would be desirable. Inhibition of caspase-3, an executioner caspase preceding DNA fragmentation, has shown to provide only moderate protection (7), and there is also a concern that blocking caspase-3 may lead to impairment of brain development because of its critical role in physiological apoptosis. Caspase-2 (Casp2) is an initiator caspase, a key enzyme in the regulation of mitochondrial permeabilization (8-10) and, therefore, theoretically a potential target for a pharmacological strategy to prevent HI brain damage. Casp2 activity has been shown to increase after HI in immature as opposed to adult mice (8). Several studies, both in vitro and in vivo, have indicated that inhibition of Casp2 is neuroprotective (9-11). Casp $2^{-1-}$ mice, as opposed to caspase $-3^{-1-}$ mice (12), do not have enlarged brains with an overabundance of neurons resulting from lack of apoptosis $(13,14)$, suggesting that interventions directed at Casp2 may not interfere with brain development.

The aim of this study was to explore the neuroprotective efficacy of hypothermia in combination with Casp2 gene deficiency in a model of neonatal $\mathrm{HI}$ in mice.

\section{RESULTS}

\section{Casp2 Gene Deletion Protects Against HI Brain Injury}

$\mathrm{HI}$ brain injury, as assessed with neuropathological score, was significantly lower in Casp $2^{-1-}$ mice as compared with wildtype (WT) mice (Kruskal-Wallis test $P<0.0001$; Figure 1a). Brain injury in WT mice was more pronounced in the hippocampus than in the striatum, cerebral cortex, and thalamus.

\footnotetext{
'Department of Neuroscience and Physiology, Sahlgrenska Academy at University of Gothenburg, Gothenburg, Sweden; ${ }^{2}$ Department of Obstetrics and Gynecology, Sahlgrenska University Hospital, Gothenburg, Sweden; ${ }^{3}$ Inserm, U676, Paris, France; ${ }^{4}$ Centre for the Developing Brain, Imperial College, Hammersmith Campus, London, UK; ${ }^{5}$ Faculté de Médecine Paris, Université Paris Diderot, Paris, France; ${ }^{6}$ Department of Child Health, University of Bristol, Bristol, UK; ${ }^{7}$ Department of Physiology, Institute of Basic Medical Sciences, University of Oslo, Oslo, Norway. Correspondence: Ylva Carlsson (ylva.carlsson@vgregion.se)
} 

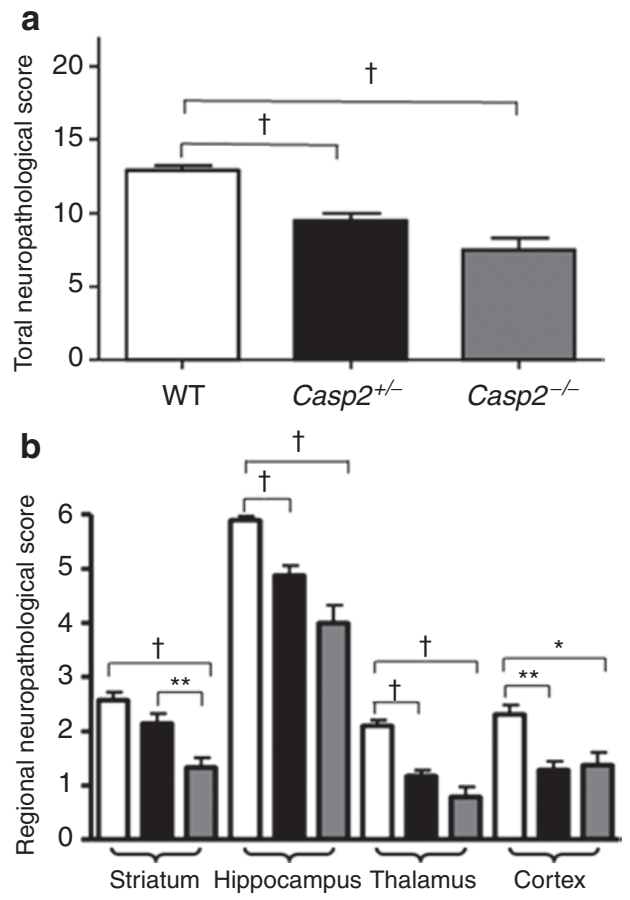

Figure 1. Brain injury after hypoxia-ischemia (HI) in mixed litters. (a) Total neuropathological score evaluated in wild-type (WT), Casp2 ${ }^{+/-}$, and Casp2 $2^{-1-}$ mice 7 days after HI. Brain injury was reduced significantly in WT vs. Casp2 $2^{+-}$as well as in WT vs. Casp2 $2^{-1-}$ mice. (b) Regional neuropathological score in the striatum, hippocampus, thalamus, and cerebral cortex:WT (open bars), Casp2 $2^{+-}$(black bars), and Casp2 $2^{-/}$(gray bars). The neuropathology score was reduced in all four regions in Casp2 $2^{-1-}$ mice as compared with WT mice. Brain injury was decreased in Casp2 $2^{+/-}$vs. WT in the hippocampus, thalamus, and cerebral cortex, whereas striatum injury was less in $\mathrm{Casp2}^{+/-}$vs. Casp2 $2^{-1-}$. Data were analyzed with Kruskal-Wallis, and values are expressed as means \pm SEM. Results from Dunn's post hoc test are given in the figure; ${ }^{\dagger} P<0.001 ;{ }^{* *} P<0.01 ;{ }^{*} P \leq 0.05$.

Brain damage was significantly reduced in both $\operatorname{Casp}^{+/-}$and Casp $2^{-/-}$mice as compared with WT mice in the hippocampus, thalamus, and cerebral cortex. In the striatum, brain injury was lower in Casp2 $2^{-/-}$mice as compared with both WT and Casp2 $2^{+/-}$ mice (Figure 1b). The neuroprotection offered by Casp2 gene deficiency was not sex dependent (total neuropathological score Casp2 $2^{-/-}$female $7.3 \pm 1.1$, Casp2 $2^{-/-}$male $7.7 \pm 1.2, \mathrm{WT}$ female $13.1 \pm 0.4$, WT male $12.6 \pm 0.5$ )

\section{Combined Effect of Casp2 Gene Deletion and Hypothermia on HI Brain Injury}

The sentinels' average temperature was $36^{\circ} \mathrm{C}$ in the normothermic chamber and $33^{\circ} \mathrm{C}$ in the hypothermic chamber. The target temperature was reached within $15 \mathrm{~min}$ for normothermic sentinels and within $5 \mathrm{~min}$ for hypothermic sentinels (Figure 2a). Five hours of hypothermia, induced immediately after HI, provided additional protection in mice with Casp2 gene deletion as compared with WT mice (two-way ANOVA, genotype $P=0.003$, temperature $P=0.0004$; Bonferroni correction, Casp $2^{-/-}$normothermia vs. Casp2 $2^{-/-}$hypothermia $P<0.01$ and Casp $^{-1-}$ hypothermia vs. WT hypothermia $P<$ $0.05)$. Total neuropathological brain injury score was reduced from $12.4 \pm 0.5$ in normothermic WT mice to $6.9 \pm 0.9$ in
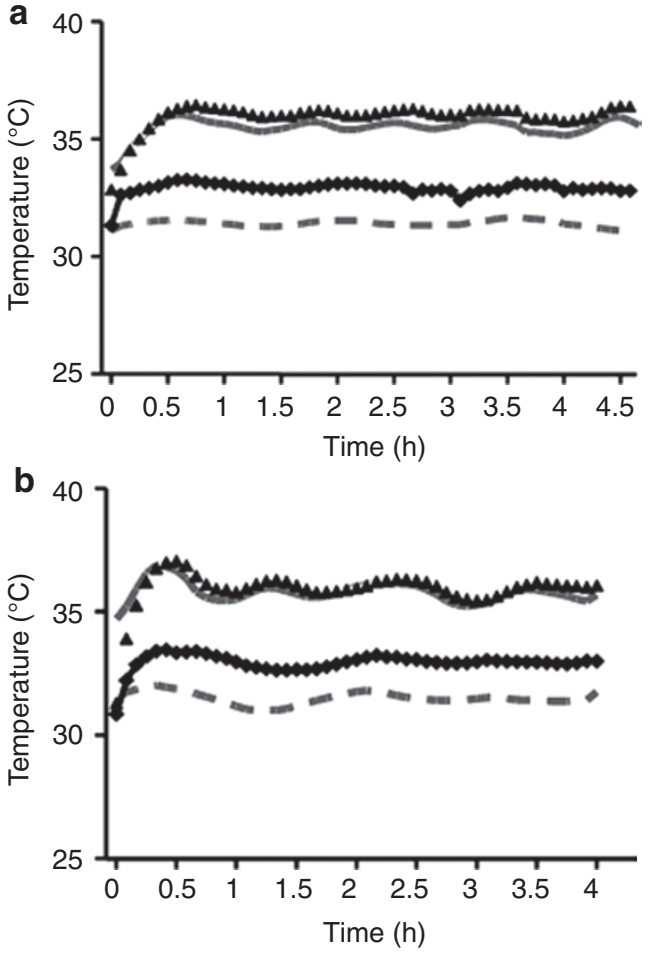

Figure 2. Temperature chart. Mean temperature during (a) $5 \mathrm{~h}$ of hypothermia/normothermia induced immediately after hypoxia-ischemia (HI) and during (b) $4 \mathrm{~h}$ hypothermia/normothermia delayed $2 \mathrm{~h}$ after $\mathrm{HI}$. One probe in each chamber monitored the environmental temperature and one animal in each chamber (hypothermia as well as normothermia) was used as sentinel for measurement of core temperature using a rectal probe. Graphs show mean environmental temperature for both chambers as well as mean rectal temperature for sentinels. Target temperatures were $36^{\circ} \mathrm{C}$ for the normothermic animals and $33^{\circ} \mathrm{C}$ for the hypothermic animals. Chamber: normothermia (gray line), hypothermia (gray dashed line); sentinel: normothermia (filled triangle), hypothermia (filled diamond).

hypothermic Casp2 $2^{-/-}$mice, whereas normothermic Casp2 $2^{-/-}$ mice had a total injury score of $10.5 \pm 1.0$ and hypothermic WT mice had a total injury score of $10.0 \pm 0.6$. Regional score demonstrated that hypothermia enhanced the protective effect of Casp2 gene deletion in the hippocampus and thalamus (Figure 3b,c). Such an additive effect was not found in the other two regions; the striatum (Figure 3a) was protected by Casp2 gene deficiency, whereas the cerebral cortex (Figure 3d) was rescued by hypothermia. White matter injury, assessed on the basis of myelin basic protein (MBP; Figure 4a) and neurofilament (NF; Figure 4b) immunoreactivity, was reduced by hypothermia independent of Casp2 genotype (two-way ANOVA; MBP: genotype $P=0.948$, temperature $P<0.0001$; NF: genotype $P=0.764$, temperature $P=0.0003)$. Furthermore, the ratio of oligodendrocyte transcription factor-2 (Olig-2)positive oligodendroglial cells in the subcortical white matter was increased following hypothermia (temperature $P=0.017$ ) but not Casp2 $2^{-/-}$(genotype $P=0.925$; WT normothermia 1.30 \pm 0.09 , WT hypothermia $1.59 \pm 0.10$, Casp $2^{-/-}$normothermia $1.39 \pm 0.08$, Casp $^{-1-}$ hypothermia $\left.1.52 \pm 0.07\right)$. We did not find a statistically significant interaction with the number of animals used in the two-way ANOVA analysis. 

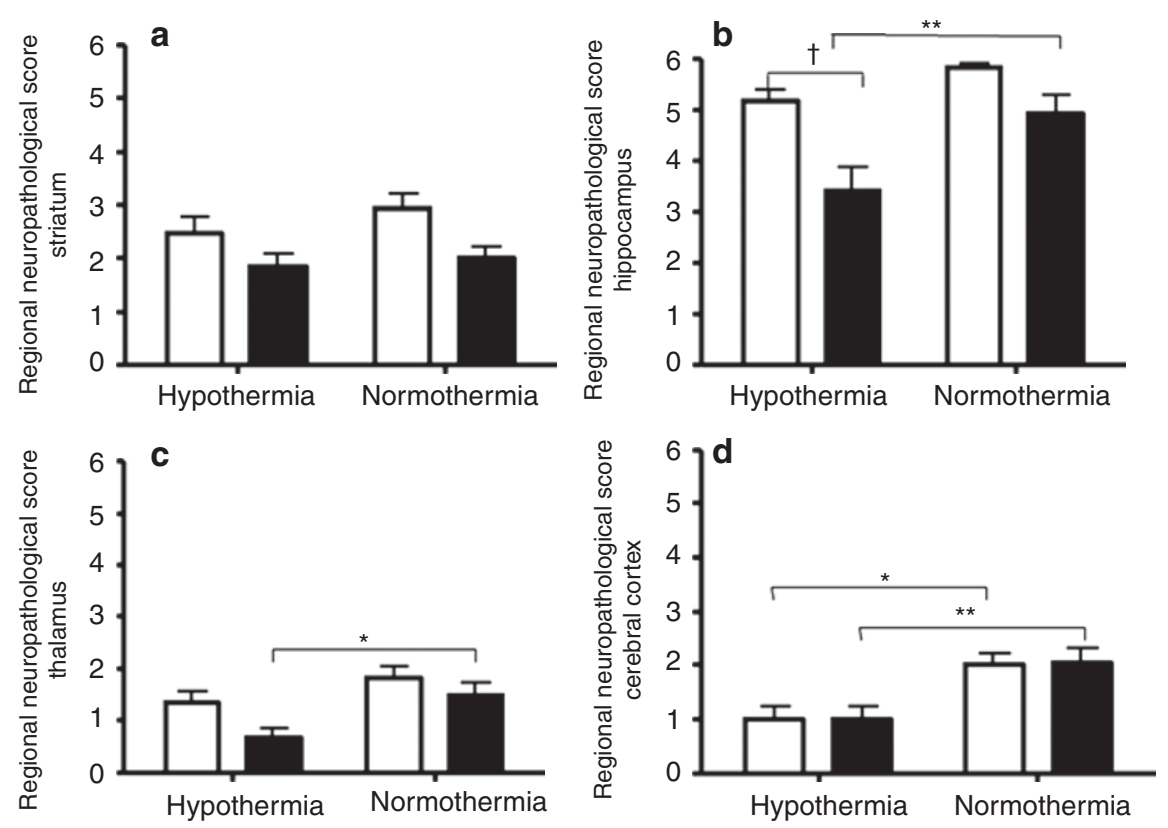

Figure 3. Regional hypoxia-ischemia (HI) brain injury in wild-type (open bars) and Casp2 $2^{--}$(filled bars) mice under hypothermic and normothermic conditions. Neuropathological score evaluated 7 days post-HI in the (a) striatum, (b) hippocampus, (c) thalamus, and (d) cerebral cortex. Hypothermia enhanced the neuroprotection offered by Casp2 gene deficiency significantly in the hippocampus (temperature $P=0.0020$, genotype $P<0.0001$ ) and the thalamus (temperature $P=0.0045$, genotype $P=0.0265$ ). The striatum was protected by Casp 2 gene deficiency (temperature $P=0.264$, genotype $P=0.0049$ ), whereas the cerebral cortex (temperature $P<0.0001$, genotype $P=0.934$ ) was rescued by hypothermia. Data were analyzed with two-way ANOVA and values are expressed as means \pm SEM. Results from Bonferroni post hoc test are given in the figure; ${ }^{+} P<0.001,{ }^{* *} P<0.01 ;{ }^{*} P \leq 0.05$.

a

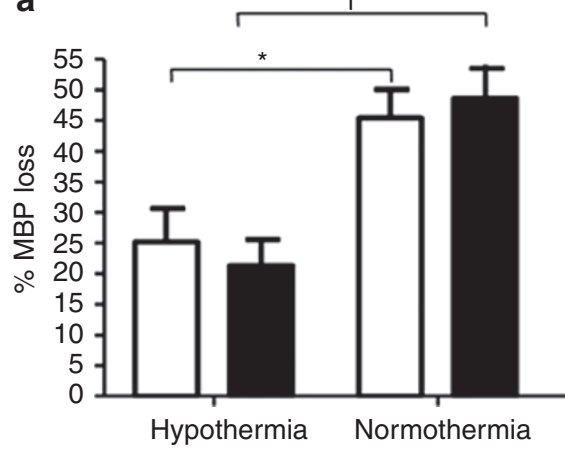

b

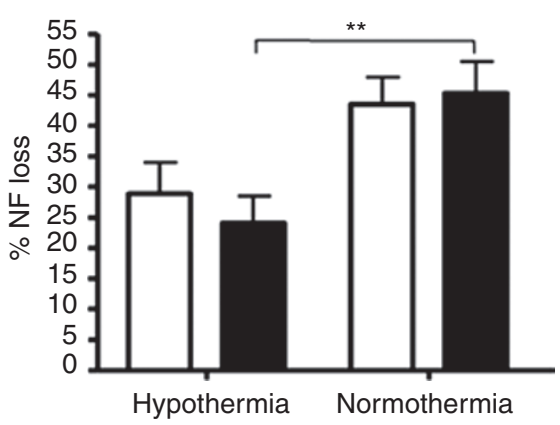

Figure 4. White matter injury after hypoxia-ischemia (HI) followed by $5 \mathrm{~h}$ of hypothermia or normothermia in wild-type (open bars) and Casp2 $2^{---}$(filled bars) mice. White matter injury measured as percentage loss of (a) MBP or (b) NF staining in the ipsilateral hemisphere in relation to the contralateral hemisphere. Injury was evaluated 7 days post-HI. Hypothermia alone decreased white matter injury, but Casp2 gene deletion did not provide additional neuroprotection (MBP: temperature $P<0.0001$, genotype $P=0.9483$; NF: temperature $P=0.0003$, genotype $P=0.4995$ ). Data were analyzed with twoway ANOVA and values are expressed as means \pm SEM. Results from Bonferroni post hoc test are given in the figure; ${ }^{\dagger} P<0.001,{ }^{* *} P<0.01 ;{ }^{*} P \leq 0.05$. MBP, myelin basic protein; NF, neurofilament.

\section{Delayed Hypothermia Protects Against HI Brain Injury}

The target temperature was reached within $10 \mathrm{~min}$ for both normothermic $\left(36^{\circ} \mathrm{C}\right)$ and hypothermic sentinels $\left(33^{\circ} \mathrm{C}\right.$; Figure $2 \mathrm{~b})$. Hypothermia treatment, delayed by $2 \mathrm{~h}$, reduced overall injury (temperature $P=0.049$, genotype $P=0.192$, post hoc Casp2 normothermia vs. Casp2 hypothermia $P<0.05$ ), as well as cerebrocortical (temperature $P=0.001$, genotype $P=0.059$, post hoc Casp2 normothermia vs. Casp2 hypothermia $P<0.01$ ) and thalamic (temperature $P=0.03$, genotype $P=0.056$, post hoc Casp2 normothermia vs. Casp2 hypothermia $P<0.05$ ) injury. Delayed hypothermia offered additive protection with Casp2 gene deletion in the hippocampus (temperature $P=0.167$, genotype $P=0.004$, post hoc WT vs. Casp2 hypothermia $P<0.01$; Figure 5) but not in the other regions. Hypothermia reduced white matter injury as assessed by MBP (temperature $P=0.002$, genotype $P=0.457$, post hoc Casp2 normothermia vs. Casp2 hypothermia) and NF (temperature $P=0.027$, genotype $P=0.335$, post hoc not significant) staining, but no additive effect with Casp2 gene deficiency was detected. There was no significant difference in Olig-2 staining between WT and Casp $2^{-/-}$mice after delayed hypothermia (data not shown). 


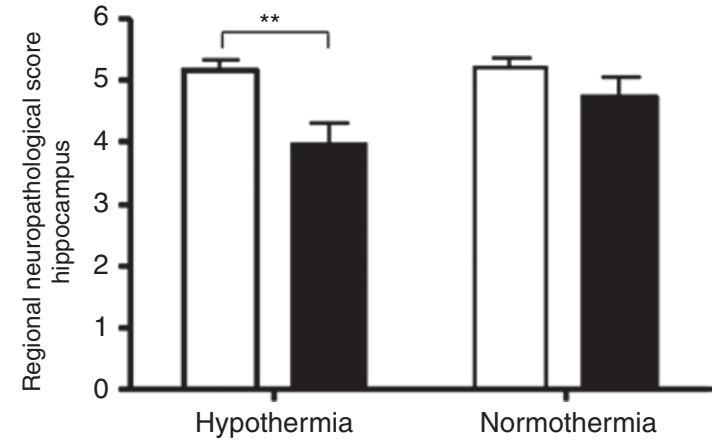

Figure 5. Brain injury after delayed hypothermia. Neuropathological score in the hippocampus of wild-type (open bars) and Casp2 $2^{--}$(filled bars) mice exposed to $4 \mathrm{~h}$ of hypothermia or normothermia delayed by $2 \mathrm{~h}$ after hypoxia-ischemia. Delayed hypothermia offered protection with Casp2 gene deletion in the hippocampus (temperature $P=0.167$, genotype $P=0.0044$ ). Data were analyzed with two-way ANOVA and values are expressed as means \pm SEM. Results from Bonferroni post hoc test are given in the figure; ${ }^{* *} P<0.01$.

\section{DISCUSSION}

This report shows that Casp $2^{-/-}$gene deletion in combination with hypothermia reduces neonatal brain injury. Total brain injury score was reduced to $6.9 \pm 0.9$ in hypothermic Casp $2^{-1-}$ mice as compared with $12.4 \pm 0.5$ in normothermic WT mice. Pharmacological treatment with xenon, erythropoietin, and topiramate has shown additional effect in combination with hypothermia, and for xenon this effect was additive, which supports the hypothesis that neuroprotection offered by hypothermia can be further improved (15-17). Our data show that hypothermic protection can be enhanced if combined with gene deficiency in a neonatal mouse model of HI. Our results agree with previous reports showing that Casp2 antisense oligonucleotides and Casp2specific small-interfering RNA, as well as Casp $2^{-/-}$cortical neurons, increase neuronal resistance in vitro to mitochondrial oxidative stress $(10,18)$ and nerve growth factor deprivation (9) and in vivo after experimental ischemic optic neuropathy in the rat (19). Furthermore, Casp2 deficiency was recently demonstrated to provide protection after $\mathrm{HI}$ as well as excitotoxic injury and Casp2-specific small-interfering RNA provided protection after excitotoxic injury (11). However the effect of Casp2 gene deficiency in the normothermic group, in the second part of the study, was less than in the within-litter experiments (Figure 1) or in our previous study (11).

The hypothesis was that the combination of hypothermia with Casp2 deletion would be superior to any intervention alone because complementary mechanisms are targeted. Hypothermia reduces secondary energy failure and mitochondrial dysfunction and attenuates the release of excitatory amino acids and free radicals, leading to a reduction in injury (20). Hypothermia has also been shown to suppress apoptosis, calcium toxicity, and the immune response $(21,22)$. Casp2 inhibition reduces mitochondrial permeabilization and activation of effector caspases (23), a key step in execution of apoptotic cell death (2).
After $5 \mathrm{~h}$ of hypothermia, an additive effect was seen not only with reference to overall injury but also regional injury in the hippocampus and thalamus, whereas the striatum was protected only by Casp2 gene deficiency and the cerebral cortex only by hypothermia. It is interesting to observe that some regions were better protected by one mode of treatment, probably related to different mechanistic profiles, and that the combination might improve the overall efficacy. Hypothermia has been shown to strongly reduce injury in the cerebral cortex as well as in the hippocampus (24), and apoptotic mechanisms seem to have different importance in different regions $(25,26)$. The lack of additive effect in some regions may well be due to partly overlapping neuroprotective mechanisms, e.g., both Casp2 gene deficiency and hypothermia improve mitochondrial integrity and attenuate downstream apoptotic mechanisms (10,21-23).

Both gray and white matter are injured by HI (1). Although mice have substantially less white matter as compared with human infants, we found a decrease in white matter injury after hypothermia, measured as loss of MBP and NF staining, and we also found that hypothermia decreased the loss of Olig2-positive cells after HI. These data agree with previous publications showing that hypothermia protects white matter (27).

The degree of protection provided by Casp2 gene deficiency varies in the different studies that we have performed. This may partly relate to the neonatal HI model, which is variable with regard to the extent of injury within and between litters (28). Secondly, in the first experiment, we used litters after mixed breeding (heterozygote (het) $\times$ homozygote), which allows comparison between littermates. Such a design cannot easily be applied when an additional treatment is added to the protocol. Hence, in the combined Casp2/hypothermia experiments, we shifted to breeding homozygote $\times$ homozygote, which may have been suboptimal at least with regard to estimation of the full contribution of the Casp2 genotype. Thirdly, the experiment involving $\mathrm{HI}$ followed by $5 \mathrm{~h}$ of hypothermia or normothermia showed a higher injury score than the two other experiments, which may have limited the protective capacity of the treatments. In our view, it is more difficult to obtain a protective effect if the injury is extensive, in agreement with experience from clinical trials (29). Finally, it was reported that Casp2 $2^{-1-}$ mouse neonates (postnatal day 1 ) develop a compensatory cell death pathway in the brain involving Smac/Diablo, endogenous inhibitors of apoptosis, leading to decreased inhibition of caspase-9, a threefold upregulation of caspase-9, and ultimately caspase-9-dependent cell death $(30,31)$. Such a compensatory mechanism could of course be at play to a variable degree also in postnatal day 9 mice, explaining why the protection offered by Casp 2 gene deficiency varied from substantial to mild/ moderate (11) in the various studies that we have completed.

In most clinical trials, cooling was started $\sim 4.5 \mathrm{~h}$ after birth (3). To mimic the clinical situation, we chose to look at delayed treatment as well. We found that a delayed start, by $2 \mathrm{~h}$, of hypothermic treatment was still effective, with an overall significant temperature effect $(P=0.049)$ and a decrease in total brain injury score by $26 \%$ in hypothermic 
Casp $2^{-1-}$ mice as compared with normothermic WT mice. However, regional evaluation showed a significant additive effect of Casp2 gene deficiency in delayed hypothermia only in the hippocampus.

Length of treatment and degree of hypothermia were chosen based on previous experiments in neonatal rats $(32,33)$. To our knowledge, this represents the first hypothermia study in a neonatal mouse model of HI. Our results of a $20 \%$ reduction in injury due to hypothermia in WT animals and no significant difference in WT animals after delayed hypothermia could indicate that the treatment modality was too mild. However, we did achieve a $45 \%$ reduction of injury after hypothermia as well as a $26 \%$ reduction in injury after delayed hypothermia in Casp2 $2^{-1-}$ mice as compared with WT normothermic mice. Zhu et al. (34) found that a minor lowering of body temperature to $34^{\circ} \mathrm{C}$ during $\mathrm{HI}$ in mice provided complete protection in the cerebral cortex. Due to a smaller body volume we expected mice to be more vulnerable than rats, which was the rationale behind using a protocol of moderate hypothermia in this study.

The average time to reach the targeted temperature was between 10 and $15 \mathrm{~min}$ in our experiments, which is comparable with hypothermia experiments in rats (35), where 20-30 min was required to reach a target core temperature of $33^{\circ} \mathrm{C}$. Sentinel animals underwent HI just as the other experimental animals to make sure that they did not temperature regulate differently than the rest. Casp $2^{-1-}$ mice and WT mice did not differ in mean temperature during hypoand normothermic conditions. Due to the fact that sentinel animals might experience increased stress because of the rectal probe; these animals were used only as temperature sentinels and excluded from the rest of the study. Even if the probe diameter was small and precautions were taken to reduce the trauma, the stress involved has been shown to affect outcome (32). Rectal temperature has been proven by several investigators to correlate well with brain temperature $(33,35)$. The temperature variation is $<0.2^{\circ} \mathrm{C}$ between individuals (36), which justifies the use of only one sentinel per chamber.

In our study, pups were separated from their dams for a prolonged time period (4-6h). Hypoglycemia might be detrimental to the immature brain after HI. However, it has been shown that blood glucose and lactate levels in experimental immature rats did not differ from those in controls after $10 \mathrm{~h}$ of separation from the dam (37). Due to the experimental conditions and the long period away from the dam an increase in mortality among the pups could have been expected; but the mortality was not higher in our experiments than the $10 \%$ reported by Thoresen et al. (36).

Hypothermia applied after HI contributed additional attenuation of injury in Casp $2^{-/-}$mice and the combination provided an additive effect rescuing more brain tissue than either intervention alone. These data support the concept that Casp2 inhibition in combination with hypothermia may have potential as a neuroprotective strategy, but more experimental work is required.

\section{METHODS}

\section{Induction of $\mathrm{HI}$ in Neonatal Mice}

In the first experiment, het Casp2 mice (Casp2 $2^{+/-}$C57BL/6; Columbia University) were bred and littermates of Casp $2^{-1-}$, het, and WT mice were exposed to HI. In the second experiment, Casp $2^{-1-}$ and WT mice were bred homozygote $\times$ homozygote. Casp $2^{-1-}$ and WT mice were, 5 min after HI, exposed to either 5 h of hypothermia $\left(33^{\circ} \mathrm{C}\right)$ or normothermia $\left(36^{\circ} \mathrm{C}\right)$. In the third experiment, $\operatorname{Cas} p 2^{-1-}$ and WT mice, bred homozygote $\times$ homozygote, were left to rest with their dams for $2 \mathrm{~h}$ after $\mathrm{HI}$ and then exposed to either $4 \mathrm{~h}$ of hypothermia $\left(33^{\circ} \mathrm{C}\right)$ or normothermia $\left(36^{\circ} \mathrm{C}\right)$. In all three experiments, unilateral $\mathrm{HI}$ was induced in mice on postnatal day 9 (28), which is approximately equivalent to human brain maturation at near-term (38-40). The left carotid artery was permanently ligated, pups were then allowed to rest for $1 \mathrm{~h}$ with their dams, before $\mathrm{HI}\left(10 \%\right.$ oxygen in nitrogen for $50 \mathrm{~min}$ at $36^{\circ} \mathrm{C}$ environmental temperature). All animal experiments were approved by the Animal Ethical Committee of Göteborg (approval 62-2008 and 172-2009). One probe in each chamber monitored the environmental temperature and one animal in each chamber (hypothermia as well as normothermia) was used as a temperature-monitoring mouse (sentinel) for measurement of core temperature using a rectal probe (T21, $0.41 \mathrm{~mm}$ diameter; Physitemp Instruments, Clifton, NJ). Computer Software Daisy lab 10.0 (Physitemp Instruments) was used to monitor the temperature with a monitor frequency of $1 / \mathrm{s}$. In the experiment with mixed litters, the mortality rate was $6 \%$, which was equivalent to the hypothermic group in the second experiment. In the normothermic group, in the second experiment, the mortality rate was $12-15 \%$, whereas with delayed hypothermia the mortality rate was $10 \%$. No difference in mortality between $\operatorname{Casp}^{2^{-1-}}$ and WT animals was detected.

\section{Gray and White Matter Injury Evaluation}

Pups (het $\times$ homozygote): Casp $2^{-1-}(n=24)$; het $(n=52)$; WT $(n=19) ; 5$ h of hypothermia (homozygote $\times$ homozygote): Casp $2^{-1-}$ normothermia $(n=25)$; Casp $2^{-1-}$ hypothermia $(n=27)$; WT normothermia $(n=22)$; WT hypothermia $(n=21)$; delayed hypothermia (homozygote $\times$ homozygote): Casp $2^{-/-}$normothermia $(n=25)$; Casp $2^{--}$hypothermia $(n=28)$; WT normothermia $(n=20)$; WT hypothermia $(n=19)$ were killed at 7 days post-HI and perfused intracardially with $\mathrm{NaCl}(0.9 \%)$ followed by $5 \%$ buffered formaldehyde (Histofix; Histolab, Göteborg, Sweden). After dehydration with graded ethanol and xylene, brains were paraffin-embedded and serially cut into $9-\mu \mathrm{m}$ coronal sections. Every 100 th section was stained. To evaluate brain injury, sections were treated according to the method described elsewhere (28) and the following primary antibodies, dilutions, and incubation times were used: mouse-antimicrotubule-associated protein-2 (MAP-2, 1:2,000, M4403, $1 \mathrm{~h}$ at room temperature; Sigma-Aldrich, St Louis, MO), mouse-anti$\operatorname{MBP}\left(1: 10,000\right.$, Smi-94, overnight at $4^{\circ} \mathrm{C}$; Covance, Princeton, NJ), mouse-anti-NF (1:2,000, Smi-312, overnight at $4^{\circ} \mathrm{C}$; Covance), and rabbit-anti-Olig-2 (1:1,000, AB9610, overnight at $4^{\circ} \mathrm{C}$; Millipore, Billerica, MA). Nonspecific labeling was investigated by omitting the primary antibody. Brain injury in different regions (striatum, hippocampus, thalamus, and cerebral cortex) was estimated on MAP2-stained sections using a semiquantitative neuropathological score system (28) and using the Olympus Micro Image analysis software system, version 4.0 (Olympus Optical, Tokyo, Japan). Subcortical white matter injury was analyzed by quantitative measurements of immunohistochemical-positive staining for MBP and NF using Micro Image, version 4.0 (Micro-Macro, Gothenburg, Sweden). The proportion of immunoreactivity in the ipsilateral hemisphere was calculated as percentage of the staining in the contralateral hemisphere. Oligodendrocyte expression was assessed by Olig-2, a marker of oligodendrocytes throughout their lineage. Images from brain sections stained with anti-Olig-2 were captured by a Leica DM 6000B camera and analyzed using Stereoinvestigator 7 (MicroBrightField System, Williston, VT). All cells within a preoutlined area, the same for all sections, in the subcortical white matter were counted, both in the ipsilateral hemisphere as well as in the contralateral hemisphere. The number of positive cells was expressed as cells $/ \mu \mathrm{m}^{2}$ and 
the number of cells in the ipsilateral hemisphere was divided by the number of cells in the contralateral hemisphere.

\section{Statistics}

Data are presented as \pm SEM and were first analyzed with the Kolmogorov-Smirnov normality test. When the data passed the normality test, the appropriate parametric test was applied (Student's $t$-test or ANOVA followed by post hoc test (Bonferroni test)). When the data did not pass the normality test, the appropriate nonparametric test was applied (Mann-Whitney or Kruskal-Wallis tests) followed by post hoc test (Dunn's test).

\section{ACKNOWLEDGMENTS}

We are very grateful to Carol Troy at Columbia University for supplying us with the $\mathrm{Casp2}^{-1-}$ mice.

\section{STATEMENT OF FINANCIAL SUPPORT}

This work was supported by a Medical Research Council strategic award (United Kingdom, P19381 to H.H.), the Medical Research Council (Sweden, 2009-2642 to H.H., K2009-54X-21119-01-4 to X.W., and 20092630 to C.M.), ALF-LUA (Sweden, ALFGBG2863 to H.H. and ALFGBG142881 to C.M.), Leducq Foundation (H.H., P.G., and C.M.), Wellcome Trust (Programme Grant WT094823MA to H.H., P.G., and E.J.), the European Union (HEALTH-F2-2009-241778, Neurobid to C.M.), Åhléns Foundation (C.M.), the Martina and Wilhelm Lundberg Foundation (Y.C.), SU-Foundation (Y.C.), and funds from the Göteborg Medical Society (to Y.C.) and the foundation for the memory of Anna Brita och Bo Castegren.

\section{REFERENCES}

1. du Plessis AJ, Volpe JJ. Perinatal brain injury in the preterm and term newborn. Curr Opin Neurol 2002;15:151-7.

2. Hagberg H, Mallard C, Rousset CI, XiaoyangWang. Apoptotic mechanisms in the immature brain: involvement of mitochondria. J Child Neurol 2009;24:1141-6.

3. Azzopardi DV, Strohm B, Edwards AD, et al. Moderate hypothermia to treat perinatal asphyxial encephalopathy. N Engl J Med 2009;361: 1349-58.

4. Edwards AD, Brocklehurst P, Gunn AJ, et al. Neurological outcomes at 18 months of age after moderate hypothermia for perinatal hypoxic ischaemic encephalopathy: synthesis and meta-analysis of trial data. BMJ 2010;340:c363.

5. Zhu C, Wang X, Xu F, et al. The influence of age on apoptotic and other mechanisms of cell death after cerebral hypoxia-ischemia. Cell Death Differ 2005;12:162-76.

6. Renolleau S, Fau S, Goyenvalle C, et al. Specific caspase inhibitor Q-VD$\mathrm{OPh}$ prevents neonatal stroke in P7 rat: a role for gender. J Neurochem 2007;100:1062-71.

7. Han $\mathrm{BH}, \mathrm{Xu} \mathrm{D}$, Choi J, et al. Selective, reversible caspase-3 inhibitor is neuroprotective and reveals distinct pathways of cell death after neonatal hypoxic-ischemic brain injury. J Biol Chem 2002;277: 30128-36.

8. Wang X, Carlsson Y, Basso E, et al. Developmental shift of cyclophilin D contribution to hypoxic-ischemic brain injury. J Neurosci 2009; 29: 2588-96.

9. Troy CM, Stefanis L, Greene LA, Shelanski ML. Nedd2 is required for apoptosis after trophic factor withdrawal, but not superoxide dismutase (SOD1) downregulation, in sympathetic neurons and PC12 cells. J Neurosci 1997;17:1911-8.

10. Chauvier D, Lecoeur H, Langonné A, et al. Upstream control of apoptosis by caspase-2 in serum-deprived primary neurons. Apoptosis 2005; 10:1243-59.

11. Carlsson Y, Schwendimann L, Vontell R, et al. Genetic inhibition of caspase-2 reduces hypoxic-ischemic and excitotoxic neonatal brain injury. Ann Neurol 2011;70:781-9.

12. Bergeron L, Perez GI, Macdonald G, et al. Defects in regulation of apoptosis in caspase-2-deficient mice. Genes Dev 1998;12:1304-14.

13. Kuida K, Haydar TF, Kuan CY, et al. Reduced apoptosis and cytochrome c-mediated caspase activation in mice lacking caspase 9. Cell 1998; 94:325-37.
14. Kuida K, Zheng TS, Na S, et al. Decreased apoptosis in the brain and premature lethality in CPP32-deficient mice. Nature 1996; 384 : 368-72.

15. Liu Y, Barks JD, Xu G, Silverstein FS. Topiramate extends the therapeutic window for hypothermia-mediated neuroprotection after stroke in neonatal rats. Stroke 2004;35:1460-5.

16. Hobbs C, Thoresen M, Tucker A, Aquilina K, Chakkarapani E, Dingley J. Xenon and hypothermia combine additively, offering long-term functional and histopathologic neuroprotection after neonatal hypoxia/ischemia. Stroke 2008;39:1307-13.

17. Cilio MR, Ferriero DM. Synergistic neuroprotective therapies with hypothermia. Semin Fetal Neonatal Med 2010;15:293-8.

18. Tiwari M, Lopez-Cruzan M, Morgan WW, Herman B. Loss of caspase2-dependent apoptosis induces autophagy after mitochondrial oxidative stress in primary cultures of young adult cortical neurons. J Biol Chem 2011;286:8493-506.

19. Ahmed Z, Kalinski H, Berry M, et al. Ocular neuroprotection by siRNA targeting caspase-2. Cell Death Dis 2011;2:e173.

20. Mueller-Burke D, Koehler RC, Martin LJ. Rapid NMDA receptor phosphorylation and oxidative stress precede striatal neurodegeneration after hypoxic ischemia in newborn piglets and are attenuated with hypothermia. Int J Dev Neurosci 2008;26:67-76.

21. Yang D, Guo S, Zhang T, Li H. Hypothermia attenuates ischemia/ reperfusion-induced endothelial cell apoptosis via alterations in apoptotic pathways and JNK signaling. FEBS Lett 2009;583:2500-6.

22. Gunn AJ, Thoresen M. Hypothermic neuroprotection. NeuroRx 2006; 3:154-69.

23. Enoksson M, Robertson JD, Gogvadze V, et al. Caspase-2 permeabilizes the outer mitochondrial membrane and disrupts the binding of cytochrome $\mathrm{c}$ to anionic phospholipids. J Biol Chem 2004;279:49575-8.

24. Wagner CL, Eicher DJ, Katikaneni LD, Barbosa E, Holden KR. The use of hypothermia: a role in the treatment of neonatal asphyxia? Pediatr Neurol 1999;21:429-43.

25. Northington FJ, Chavez-Valdez R, Martin LJ. Neuronal cell death in neonatal hypoxia-ischemia. Ann Neurol 2011;69:743-58.

26. Northington FJ, Zelaya ME, O'Riordan DP, et al. Failure to complete apoptosis following neonatal hypoxia-ischemia manifests as "continuum" phenotype of cell death and occurs with multiple manifestations of mitochondrial dysfunction in rodent forebrain. Neuroscience 2007;149:822-33.

27. Roelfsema V, Bennet L, George S, et al. Window of opportunity of cerebral hypothermia for postischemic white matter injury in the near-term fetal sheep. J Cereb Blood Flow Metab 2004;24:877-86.

28. Hedtjärn M, Leverin AL, Eriksson K, Blomgren K, Mallard C, Hagberg H. Interleukin-18 involvement in hypoxic-ischemic brain injury. J Neurosci 2002;22:5910-9.

29. Gluckman PD, Wyatt JS, Azzopardi D, et al. Selective head cooling with mild systemic hypothermia after neonatal encephalopathy: multicentre randomised trial. Lancet 2005;365:663-70.

30. Troy CM, Rabacchi SA, Hohl JB, Angelastro JM, Greene LA, Shelanski ML. Death in the balance: alternative participation of the caspase- 2 and -9 pathways in neuronal death induced by nerve growth factor deprivation. J Neurosci 2001;21:5007-16.

31. Troy CM, Friedman JE, Friedman WJ. Mechanisms of p75-mediated death of hippocampal neurons. Role of caspases. J Biol Chem 2002;277: 34295-302.

32. Thoresen M, Bågenholm R, Løberg EM, Apriccna F. The stress of being restrained reduces brain damage after a hypoxic-ischaemic insult in the 7-day-old rat. Neuroreport 1996;7:481-4.

33. Thoresen M, Bågenholm R, Løberg EM, Apricena F, Kjellmer I. Posthypoxic cooling of neonatal rats provides protection against brain injury. Arch Dis Child Fetal Neonatal Ed 1996;74:F3-9.

34. Zhu C, Wang X, Xu F, et al. Intraischemic mild hypothermia prevents neuronal cell death and tissue loss after neonatal cerebral hypoxia-ischemia. Eur J Neurosci 2006;23:387-93.

35. Trescher WH, Ishiwa S, Johnston MV. Brief post-hypoxic-ischemic hypothermia markedly delays neonatal brain injury. Brain Dev 1997;19:326-38. 


\section{Articles | Carlsson et al.}

36. Thoresen M, Hobbs CE, Wood T, Chakkarapani E, Dingley J. Cooling combined with immediate or delayed xenon inhalation provides equivalent long-term neuroprotection after neonatal hypoxia-ischemia. J Cereb Blood Flow Metab 2009;29:707-14.

37. Dingley J, Hobbs C, Ferguson J, Stone J, Thoresen M. Xenon/ hypothermia neuroprotection regimes in spontaneously breathing neonatal rats after hypoxic-ischemic insult: the respiratory and sedative effects. Anesth Analg 2008;106:916-23, table of contents.
38. Brazel CY, Rosti RT 3rd, Boyce S, Rothstein RP, Levison SW. Perinatal hypoxia/ischemia damages and depletes progenitors from the mouse subventricular zone. Dev Neurosci 2004;26:266-74.

39. Craig A, Ling Luo N, Beardsley DJ, et al. Quantitative analysis of perinatal rodent oligodendrocyte lineage progression and its correlation with human. Exp Neurol 2003;181:231-40.

40. Romijn HJ, Hofman MA, Gramsbergen A. At what age is the developing cerebral cortex of the rat comparable to that of the full-term newborn human baby? Early Hum Dev 1991;26:61-7. 